\title{
Profiling and Bioinformatics Analysis Revealing Differential Circular RNA Expression about Storage Lesion Regulatory in Stored Red Blood Cells
}

\author{
Yiyu Zhang ${ }^{a, b}$ Guoqing Huang ${ }^{a}$ Zhaohu Yuan ${ }^{b, c}$ Yonggang Zhang ${ }^{a}$ \\ Xiaojie Chen ${ }^{\text {b, c }}$ Jianyun Huang ${ }^{\text {b, } c}$ Nan Lib,c Zhen Liub,c Weijie Zhong ${ }^{b}$ \\ Huikang Huang ${ }^{b, c}$ Canze Huang ${ }^{d}$ Yaming Wei ${ }^{b, c}$ \\ ${ }^{a}$ Department of Blood Transfusion, Shenzhen Longhua District Central Hospital, Shenzhen, China; ${ }^{b}$ Department of \\ Blood Transfusion, Guangzhou First People's Hospital, School of Medicine, South China University of Technology, \\ Guangzhou, China; 'Guangdong Engineering Research Center of Precise Transfusion, Guangzhou First People's \\ Hospital, School of Medicine, South China University of Technology, Guangzhou, China; ${ }^{d}$ Department of \\ Gastroenterology, Zhuhai People's Hospital, Zhuhai Hospital affiliated with Jinan University, Zhuhai, China
}

\section{Keywords}

Stored red blood cells $\cdot$ circRNA $\cdot$ miRNA $\cdot$ ceRNA $\cdot$ Storage lesion

\begin{abstract}
Introduction: Circular RNA (circRNA) plays an important role in regulating metabolism of red blood cells (RBCs) and their storage lesions, but the study of how circRNA expression changes in stored RBCs has rarely been conducted. Methods: The expression change of circRNA was systemically evaluated via high-throughput sequencing on healthy RBCs on day 0 , 20 , and 40 . And then we confirmed the reliability of the highthroughput sequencing analysis by RT-qPCR characterization on selected circRNAs. A higher parental gene enrichment was used to explore circRNA function in pathways. In addition, we deciphered a dysregulated circRNA-related ceRNAs network, and identified three circRNA-miRNA-mRNA regulatory axes related to storage lesion. Results: We identified 2,586 known and 6,216 putative novel circRNAs, more than 100 circRNAs expression levels were shifted, and the number of downregulated circRNAs was greater with longer storage time. Furthermore, a higher parental gene enrichment related to circRNA was found in pathways, including CAMP signaling pathway, ubiquitin-mediated proteolysis, apoptosis, adhesion, MAPK signaling pathway, cystine methionine metabolism, RNA
\end{abstract}

degradation, RNA transport, TGF- $\beta$, and actin regulatory pathway. hsa_circ_0007127-miR-513a-5p-SMAD4, hsa_ circ_0000033-miR-19a-3p-VAMP3, and hsa_circ_0005546miR-4720-CCND3 regulatory axes related to storage lesion was found. Conclusions: Through investigation in circRNAs profile and circRNA-miRNA-mRNA interactions, this study provides insights on stored RBC circRNA expression changes, which closely relate to the storage lesion of RBCs and their physiological functions.

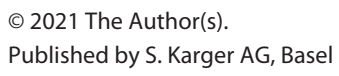

\section{Introduction}

Stored red blood cells (RBCs) undergo storage lesions in blood bank conditions that progressively affect their metabolism, cytoskeleton, and membrane [1]. Importantly, the damaged RBCs cause hemolysis, failure in cure anemia, and reduction on RBC survival rate after transfusion [2]. So far, researchers have paid little attention to systematically study RBCs storage lesion regarding the exact nature of this phenomenon and the involved molecular factors.

It is known that the precise regulation of both coding and noncoding RNAs is essential for hematopoietic stem cell differentiation to RBC. The RBC storage lesion is often

\section{karger@karger.com} www.karger.com/tmh

Karger ${ }^{\prime \prime}$

BOPEN ACCESS
(C) 2021 The Author(s)

Published by S. Karger AG, Basel

This is an Open Access article licensed under the Creative Common Attribution-NonCommercial-4.0 International License (CC BY-NC) (http://www.karger.com/Services/OpenAccessLicense), applicable to the online version of the article only. Usage and distribution for commercial purposes requires written permission.
Correspondence to:

Yaming Wei, eywym@scut.edu.cn 
accompanied by significant transcriptome changes; we previously found that 211 miRNAs were dysregulated in day 20 group compared to day 0 group [3]. As a vital participant in transcriptome changes of stored RBCs, circular RNA (circRNA) is endogenously expressed as singlestranded and always appears as a covalently closed circular molecule [4]. There are 17 to 188 times more back-splice reads of circRNAs presented in platelets than those in human nucleated tissues, and this situation also exists in erythrocyte even stored after 27 days in blood bank conditions [5]. Notably, circRNA enrichment occurs after platelet and erythrocyte loss of their nuclear influence [5]. This suggests that circRNAs are expressed at quite a high level for their alleged miRNA target to take some biological effect and even probably play an unique role in the physiological activities of anucleated cells. Therefore, for circRNAs, their abundance relative to linear RNAs and stable structure relative to miRNA are ideal potential markers for investigation of storage lesion in the storage of RBCs.

To fill the knowledge gap on the circRNA expression changes in stored RBCs, in this study, we performed circRNA high-throughput sequencing analysis on day 0,20 , and 40 for stored RBCs. In the following, different investigations including Mireap, Miranda, TargetScan, circBase, miRBase, miRTarBase software analysis and sequence alignment, were carried out to characterize the targeting relationship between circRNAs and their target miRNAs. Finally, the expression and function of target miRNAs, their downstream target mRNAs, the regulation pathways of these circRNAs, and their relationship was systematically evaluated for understand RBC storage lesion, including energy metabolism, morphological changes, and apoptosis.

\section{Methods}

\section{Blood Sample Collection and Groups}

Current studies have shown that changes in membrane redox status during blood-bank storage condition are dependent on gender and preservation solution [6]. Owing to resource limitation, in this study we selected male volunteers, in which erythrocytes from the male donors are mostly affected by free radical-mediated oxidative stress [6]. Blood from 5 healthy adult male volunteers (aged 23-30 years, blood group O) was collected in a blood polyvinyl chloride plastic bag containing anticoagulant citrate phosphate dextrose adenine solution (CPDA-1, Fresenius Kabi, Guangzhou, China). The blood samples were filtrated to remove leukocytes, and the suspended RBCs were collected and further stored at $4^{\circ} \mathrm{C}$. During the following characterization, three equal samples were used and tested on day 0,20 , and 40 , respectively. Three samples were tested during high-throughput sequencing, while RT-qPCR test was conducted for three times for all samples.

\section{RBC Purification}

Suspended fresh RBCs were leuko-reduced by use a leukocyte depletion filter (Haemonetics Manufacturing Inc, Covina, CA, USA), which can minimize potential leukocyte contamination. In each sample, a total of $4.40 \times 10^{12} / \mathrm{L}$ RBCs were tested. RBCs were additionally purified by washing with $0.9 \% \mathrm{NaCl}$ and centrifuged three times at $1,400 \times g$ for 6 min to remove platelet and plasma contamination before storage.

\section{Total RNA Extraction and circRNA Isolation}

Total RNA was extracted from RBCs with TRIzol reagent (Ambion, Life Technology, Carlsbad, CA, USA), and then treated with DNase I (Worthington Biochemical, Freehold, NJ, USA) according to the manufacturer's protocol to remove DNA contamination without purification for keeping RNA integrity. RNA purity and integrity was characterized using a NanoDrop 2000 spectrophotometer (Thermo Scientific, Waltham, MA, USA) and the RNA Nano 6000 Assay Kit of the Agilent Bioanalyzer 2100 system (Agilent Technologies, CA, USA), respectively. And then removal ribosome RNA and purification by using a Low Input RiboMinus ${ }^{\mathrm{TM}}$ Eukaryote System v2 kit (Ambion, Life Technology, Carlsbad, CA, USA). At last, using the Ribonuclease R, E. coli kit (Epicentre, Madison, WI, USA) which contains RNase $\mathrm{R}$ to digests linear RNA.

circRNA Library Construction and RBC circRNA-Seq Analysis

After RNase R digestion, a total of more than $30 \mathrm{ng}$ circRNA was used to prepare circRNA-seq libraries. The circRNA fragments about $200 \mathrm{nt}$ was enriched with the magnetic beads having Oligo(dT). Then the fragmentation buffer was added to the enriched RNA in order to obtain many short fragments that were used as a template. Following QIAquick PCR Purification kit (QIAGEN, Hilden, Germany), the first chain of cDNA was synthesized by six random hexamers, and double strand cDNA was synthesized by SuperScriptII and random primers. Furthermore, cDNA was end-repaired, adenylated, and ligated to adapters. A 120-260 nt fragment was recycled by agarose. cDNA libraries were further amplified and sequenced on Illumina HiSeqTM 4000 (Illumina, San Diego, CA, USA).

\section{Bioinformatics and Data Analysis}

Detection and clustering of putative circularization junctions are according to the protocols as follows. First, after sequencing analyses, the machine data were obtained and further filtered to get high-quality clean reads. We use FastQC (v0.11.5) to provide a simple way to do some quality control cheIDs on raw sequence data coming from high-throughput sequencing pipelines. Second, Bowtie2 (v2.2.9) was conducted to map reads to the genome of species. Unmapped reads were still mapped to the genome using tophat-fusion next. Then we de novo assembled the mapped reads to circRNAs by UROBORUS (v.0.1.3). At last, back splicing reads were identified in unmapped reads by tophat-fusion and UROBORUS (v.0.1.3). Differentially expressed circRNAs (FDR $<0.05$ and fold change $>2$ ) were annotated exhaustive with the circRNA/miRNA interaction information. CircRNA/miRNA interactions were predicted using Arraystar miRNA target prediction software based on TargetScan, Miranda, and Mireap. MirTarBase (r6.0 Sept. 2015) was used to find the reported relationship information between mRNA and miRNA. CirRNA-miRNA-mRNA interaction analysis was conducted by Cytoscape software. Several circRNA-miRNA-mRNA regulatory axes are discovered and then verified by RT-qPCR. PCR primers were designed using the online software Primer (http://frodo.wi.mit.edu/primer3/). KEEG enrichment analysis of circRNA expressed by each group allows to understand the biological function of the whole differentially expressed circRNA. KEGG is the main public database for pathway enrichment analysis and using KEGG pathway as a unit, a hypergeometric test was used to identify pathways significantly enriched in differentially expressed genes compared to the entire genomic background. KEEG and Go analysis were performed for the differential expression circRNA-associated parental genes. 
Validation of Selected circRNAs by RT- $q P C R$

CircRNAs were detected by quantitative polymerase chain reaction (PCR). To quantify relative circRNA expression, the RNA was extracted from RBC with Trizol reagent, according to the manufacturer's protocol (Life Technologies, Scotland, UK). Generally, $500 \mathrm{ng}$ of total RNA was firstly treated with RNaseR (Epicentre, catalog number: RNR07250, lot number RNR40620) for digestion, and then reverse transcribed in a final volume of $10 \mu \mathrm{L}$ using random primers and standard conditions using the Prime Script RT Master Mix (TaKaRa, Dalian, China). Then, RT-qPCR adopting $20 \mu \mathrm{L}$ reaction system, including $2 \mu \mathrm{L}$ complementary DNA (cDNA) was performed by the SYBR Premix Ex Taq Master Mix II (TaKaRa, Dalian, China) on the qTOWER2.2 (analytikjena, Jena, Germany), according to the manufacturer's instructions. $\beta$-Actin was used as the internal control. Primers were provided by the Shanghai Generay Biotech Co., Ltd and Takara Bio Inc and the sequences are listed in Table 1.

The RT-qPCR reaction included an initial denaturation step at $95^{\circ} \mathrm{C}$ for $30 \mathrm{~s}$, which was followed by 40 cycles of treatment, including $95^{\circ} \mathrm{C}$ for $5 \mathrm{~s}, 58^{\circ} \mathrm{C}$ for $30 \mathrm{~s}, 72^{\circ} \mathrm{C}$ for $30 \mathrm{~s}$, a cycle of $95^{\circ} \mathrm{C}$ for 15 $\mathrm{s}, 60^{\circ} \mathrm{C}$ for $1 \mathrm{~min}$, and $95^{\circ} \mathrm{C}$ for $15 \mathrm{~s}$. The $2^{-\Delta \Delta \mathrm{Ct}}$ method was used to calculate circRNA relative expression. See online supplementary Table 1 for primers used in RT-qPCR (for all online suppl. material, see www.karger.com/doi/10.1159/000519626).

\section{Statistical Analysis}

SPSS (version 16.0, Chicago, IL, USA) was used for statistical analyses. Experimental data were expressed as the mean \pm standard deviation. Variables of the high-throughput sequencing and RT-qPCR data between the three groups were compared by ANOVA. The criteria of fold change $>2$ and FDR $<0.05$ were used to screen differentially expressed (DE) circRNAs.

\section{Results}

\section{Expression Characteristics of all circRNAs and}

Screening of Differentially Expressed circRNAs

The sequencing quality assessment results showed that high-quality clean reads in each sample exceeded $99 \%$, confirming that the results were very credible. A total of 8,802 circRNAs were detected, composed of 2,586 known circRNAs and 6,216 novel circRNAs. The highest known circRNAs length is $2,500 \mathrm{bp}$, and most of the circRNAs we found were between $200 \mathrm{bp}$ and $300 \mathrm{bp}$ in length (Fig. 1A). Most of circRNAs transcribed from

Table 1. The information of 15 DE-circRNA/DE-miRNA

\begin{tabular}{|c|c|c|c|c|c|c|c|}
\hline Targeted miRNA & Gene ID & Source gene & Chr & Strand & Genomic start & Genomic end & annot type \\
\hline hsa-miR-1299 & hsa_circ_0011095 & ENST00000311772 & 1 & + & 28167524 & 28169841 & annot_exons \\
\hline hsa-miR-198 & hsa_circ_0008102 & ENST00000540664 & 10 & + & 98703869 & 98711953 & annot_exons \\
\hline hsa-miR-330-5p & hsa_circ_0000722 & ENST00000253458 & 16 & + & 85667519 & 85667738 & one_exon \\
\hline hsa-miR-3675-5p & hsa_circ_0000883 & ENST00000303657 & 19 & - & 6026173 & 6040252 & annot_exons \\
\hline hsa-miR-4270 & hsa_circ_0005546 & ENST00000263377 & 19 & - & 15379715 & 15383944 & annot_exons \\
\hline hsa-miR-4270 & hsa_circ_0007167 & ENST00000357681 & 1 & + & 203676136 & 203677232 & annot_exons \\
\hline hsa-miR-4419b & hsa_circ_0007127 & ENST00000552231 & 12 & + & 70671911 & 70704797 & annot_exons \\
\hline hsa-miR-4456 & hsa_circ_0008554 & ENST00000586659 & 19 & - & 11289020 & 11289396 & annot_exons \\
\hline hsa-miR-4530 & hsa_circ_0035761 & ENST00000443617 & 15 & - & 63966540 & 63981898 & annot_exons \\
\hline hsa-miR-4534 & hsa_circ_0007995 & ENST00000372979 & 10 & - & 74906033 & 74923708 & annot_exons \\
\hline hsa-miR-4645-3p & hsa_circ_0002473 & ENST00000602402 & 13 & + & 96375495 & 96377506 & annot_exons \\
\hline hsa-miR-4687-3p & hsa_circ_0000883 & ENST00000303657 & 19 & - & 6026173 & 6040252 & annot_exons \\
\hline hsa-miR-513a-5p & hsa_circ_0007127 & ENST00000552231 & 12 & + & 70671911 & 70704797 & annot_exons \\
\hline hsa-miR-676-3p & novel_circ_0002503 & ENST00000397013 & 11 & - & 3789810 & 3789910 & one_exon \\
\hline
\end{tabular}

Fig. 1. Expression characteristic of circular RNA in stored RBC. A Differential length distribution among different storage time samples. B The source composition of circRNA classified according to the source region. annot_exon: the breakpoint of a circular RNA is located at the start of one exon and the end of another exon of a known gene, and its sequence is composed of the bases of the exons between the breakpoints. one_exon: circular RNA is located in an exon of a known gene, and its sequence is composed of all bases between breakpoints. exon_intron: there are one or more complete exons of a known gene between the breakpoints of the circular RNA, but the breakpoint is not at the beginning or end of the known exon, and its sequence is from the breakpoint start site to the first base structure of exon start site, exon between breakpoint, and last exon to breakpoint stop. intronic: circular RNA is located in an intron of a known gene, and its sequence is composed of all bases between breakpoints. C Chromosome distribution of all circRNAs. Only some chromosomes were aligned with all circRNAs. D CircRNA expression density distribution. The abscissa in the figure is $\log 10$ (SRPM) (spliced reads per million mapped reads), the ordinate is the abundance of circular RNA (expression of a certain circular RNA/total expression of expressed circular RNA). The higher the value is, the higher the level of circular RNA expression will be; all samples were divided into 3 groups, day 0 (A1, B1, C1), day 20 (A2, B2, C2), and day 40 (A3, B3, C3). Each curve represents a sample. E Different circRNAs expression profiles in stored RBC.

(For figure see next page.) 
A

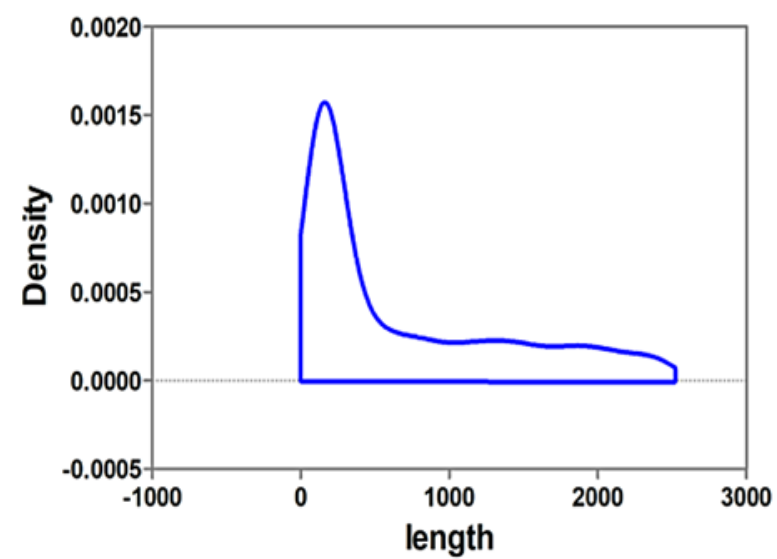

C

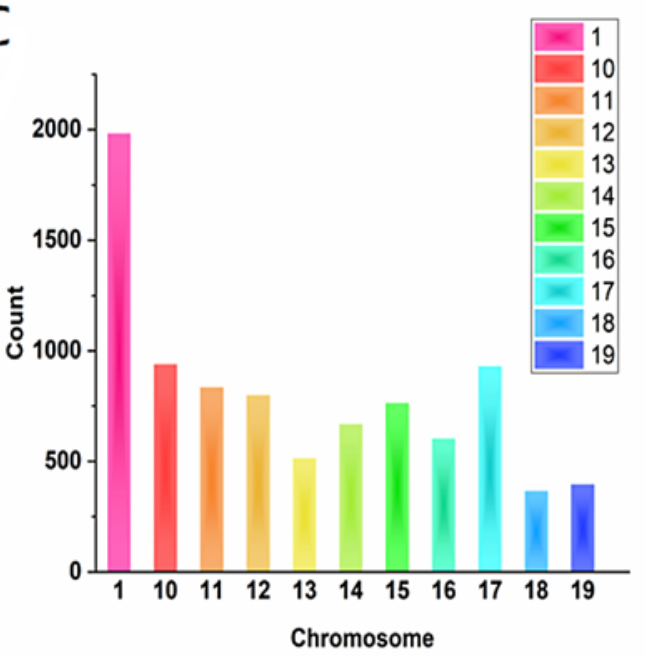

E

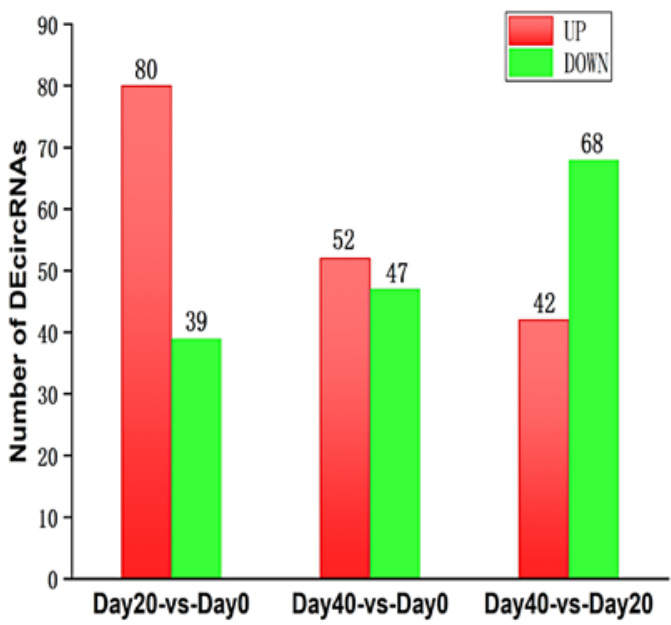

B

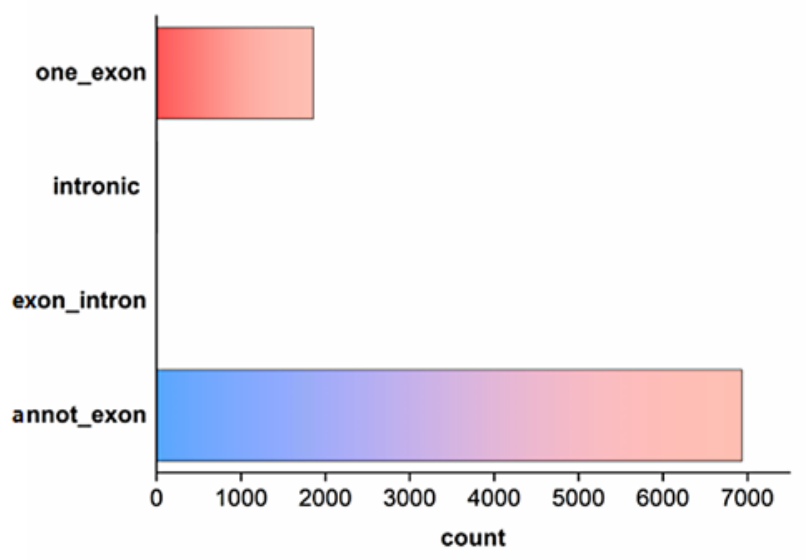

D

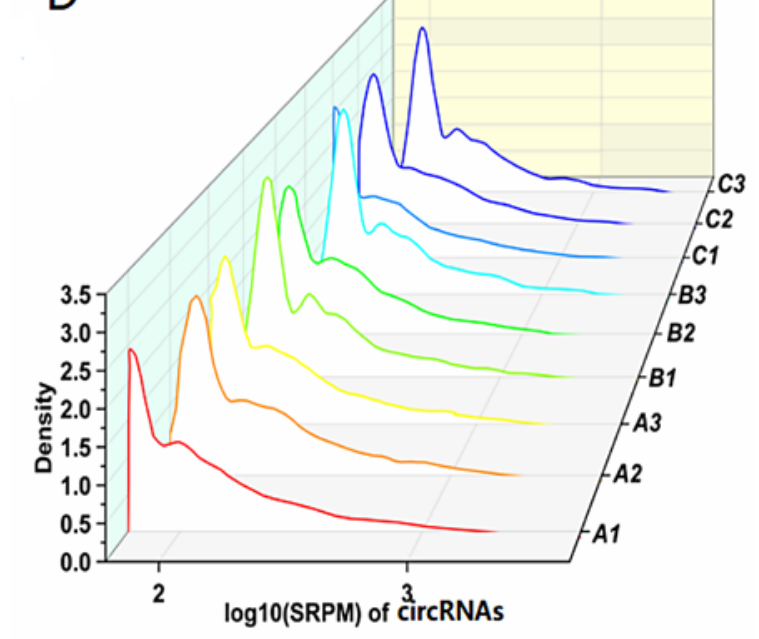

Day20-vs-Day0 Day40-vs-Day0

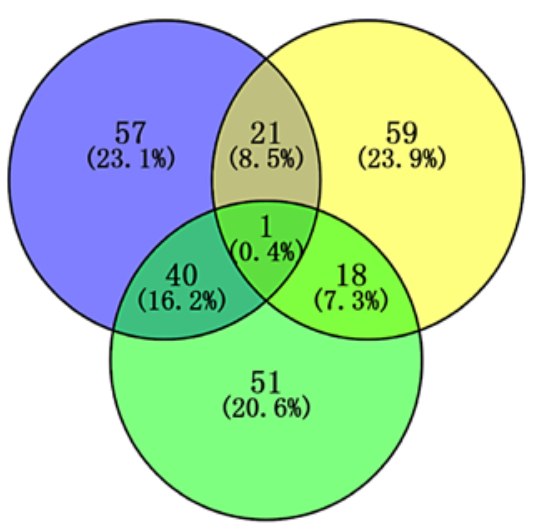

Day40-vs-Day20 
the protein coding exons and annot_exon type (the break point of a circRNA is located at the start of one exon and the end of another exon of a known gene) of circRNA has the largest proportion (Fig. 1B). The distribution of circRNAs in chromosomes might be connected with the biological processes in which the circRNAs were involved. It is because the role of circRNA largely depends on their binding targets. Figure $1 \mathrm{C}$ shows the distribution of all circRNAs located on chromosome 1, 10-19. Figure 1D shows that abundance of circular RNAs expression in each sample of the same group was closer.

According to the results for day 20 versus day 0 , it revealed that expression levels of 119 circRNAs have changed, in which 80 circRNAs were increased and 39 circRNAs were decreased. As to the results for day 40 versus day 0 , it is suggested that expression levels of 99 circRNAs have changed, in which 52 circRNAs were increased, while 47 circRNAs were decreased. In addition, for the results of day 40 versus day 20, it demonstrated that expression levels of 110 circRNAs were changed, in which 42 circRNAs were increased and 68 circRNAs were decreased (Fig. 1E). In summary, 247 circRNAs were differentially expressed with fold change $>2$ and FDR $<0.05$ by Venn diagram analysis. Notably, in day 20 vs. day 0 , day 40 vs. day 0 , and day 40 vs. day 20 , only one circRNA (hsa_circ_0000033) was differently expressed (Fig. 1F).

\section{The Differentially Expressed circRNAs Parental Gene Were Mainly Enriched in Cellular Nitrogen Compound Metabolic Process and Ubiquitin- \\ Mediated Proteolysis Pathways}

GO terms $(p<0.05)$ in all comparison groups classified by BP (biological process), CC (cellular component), MF (molecular function) and ranked by $p$ value were listed (Fig. 2). These differently expressed genes for each comparison group were subjected to GO enrichment analysis to evaluate the enrichment of genes with a certain function. Importantly, all of these responses were helpful for understanding about storage lesion events. The results showed that the term with the highest frequency in GO analysis was in line with the comparison of the three groups, just as follows. For day 20 versus day 0 and day 40 versus day 0 group, the most enriched and meaningful $\mathrm{BP}$ term was cellular nitrogen compound metabolic process. For day 40 versus day 20, the most enriched and meaningful BP term was cellular macromolecule metabolic process. As for CC, the most enriched term was intracellular. And the most enriched MF term was nucleic acid binding. Moreover, KEGG pathway analysis was conducted to select the top 20 pathways and rank the degree of gene enrichment (Fig. 3). For day 20 versus day 0 group, the dominant pathway was ubiquitin-mediated proteolysis. For day 40 versus day 0 group, the dominant pathway was protein processing in endoplasmic reticulum. Perhaps it was because the RBCs form vesicles in the cell membrane during long-term storage. For day 40 versus day 20 group, the dominant pathway was also ubiquitin-mediated proteolysis.

\section{Construction Networks of Differentially Expressed circRNAs and Their Binding miRNAs}

We used TargetScan, Mireap, and miRanda database to search for the target sites of the conserved seed-matching sequences of the DE-circRNAs and their target miRNAs and identified 246, 194, and 190 circRNA-miRNA interactions in day 20 versus day 0 , day 40 versus day 0 , and day 40 versus day 20, respectively. Notably, the DEcircRNA species, including hsa_circ_0000722, hsa circ_0000883, hsa_circ_0008554, hsa_circ_0037130, hsa_circ_0037131, hsa_circ_0004123, hsa_circ_0036351, hsa_circ_0037130, hsa_circ_0000883 and hsa circ_0046702, can sponge with at least 10 miRNAs.

An entire network of DE-circRNA/miRNA interaction is displayed as a network generated by cytoscape software (Fig. 4). Furthermore, we identified the potential targets of the miRNAs based on mirTarBase with collections of experimentally supported miRNA-target interactions. Thus, 283,366, 194,100, and 268,940 miRNAmRNA interactions were detected in day 20 versus day 0 , day 40 versus day 0 , and day 40 versus day 20 , respectively. The finding of the reported relationship between mRNAs and miRNAs assists to elucidate the biological function of circular RNAs.

We proceeded to identify potentially circRNA-miRNA-mRNA regulatory axes based on our formerly identified DE-circRNAs (day20 vs. day 0) and the DE-miRNAs (day 20 vs. day 0) [3]. It is noticed that the DE-miRNAs (109 increased and 102 decreased miRNAs) were detected from healthy male RBCs, which contributes to identifying 15 DE-circRNA-DE-miRNA interactions (Table 1).

\section{Verification of Selected circRNAs Related to Storage \\ Lesion}

According to Figure 5, it shows that selection of 17 circRNAs for RT-qPCR was consistent with the highthroughput data. The details of the selected 17 circRNAs are shown in online supplementary Table 2 . These 17 circRNAs were related to storage lesion with the reasons as follows. First, these circRNAs had stable expression and were easily detected at day 0 , day 20 , and day 40 . Second, their host linear transcripts were belonging to cell apoptosis in annotation. Most of the parental genes, which correlates to apoptosis and acid changes like ubiquitinmediated proteolysis and RNA degradation in storage lesion, were characterized by both KEGG analysis and GO analysis. Third, even if some circRNAs were not directly
Zhang/Huang/Yuan/Zhang/Chen/Huang/ Li/Liu/Zhong/Huang/Huang/Wei 


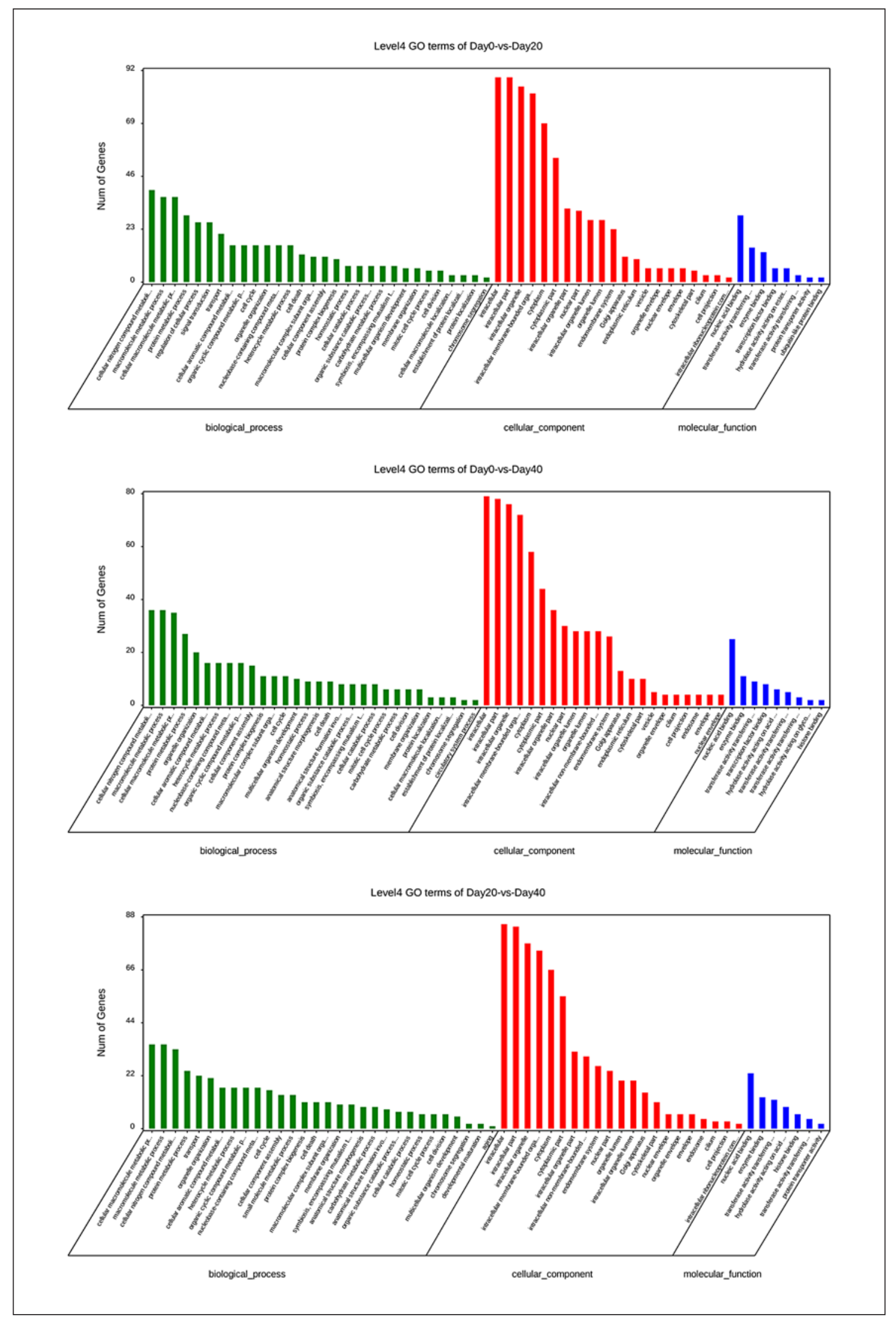

Fig. 2. Go analysis of differentially expressed circRNAs parental gene $(n=3)$. 
Fig. 3. Pathways of differentially expressed circRNAs parental gene $(n=3)$. A Day 20 versus day 0 source gene KEGG enrichment analysis bubble chart. B Day 40 versus day 0. C Day 40 versus day 20. Bubble represents the gene number and the color represents the $p$ value. For example, smallest bubble $=1$ parental gene of circRNA; largest bubble $=5-6$ parental gene of circRNAs . Rich factor: number of differentially expressed circRNA's parental gene enriched in this pathway/number of background parent gene of all circRNAs enriched in this pathway.

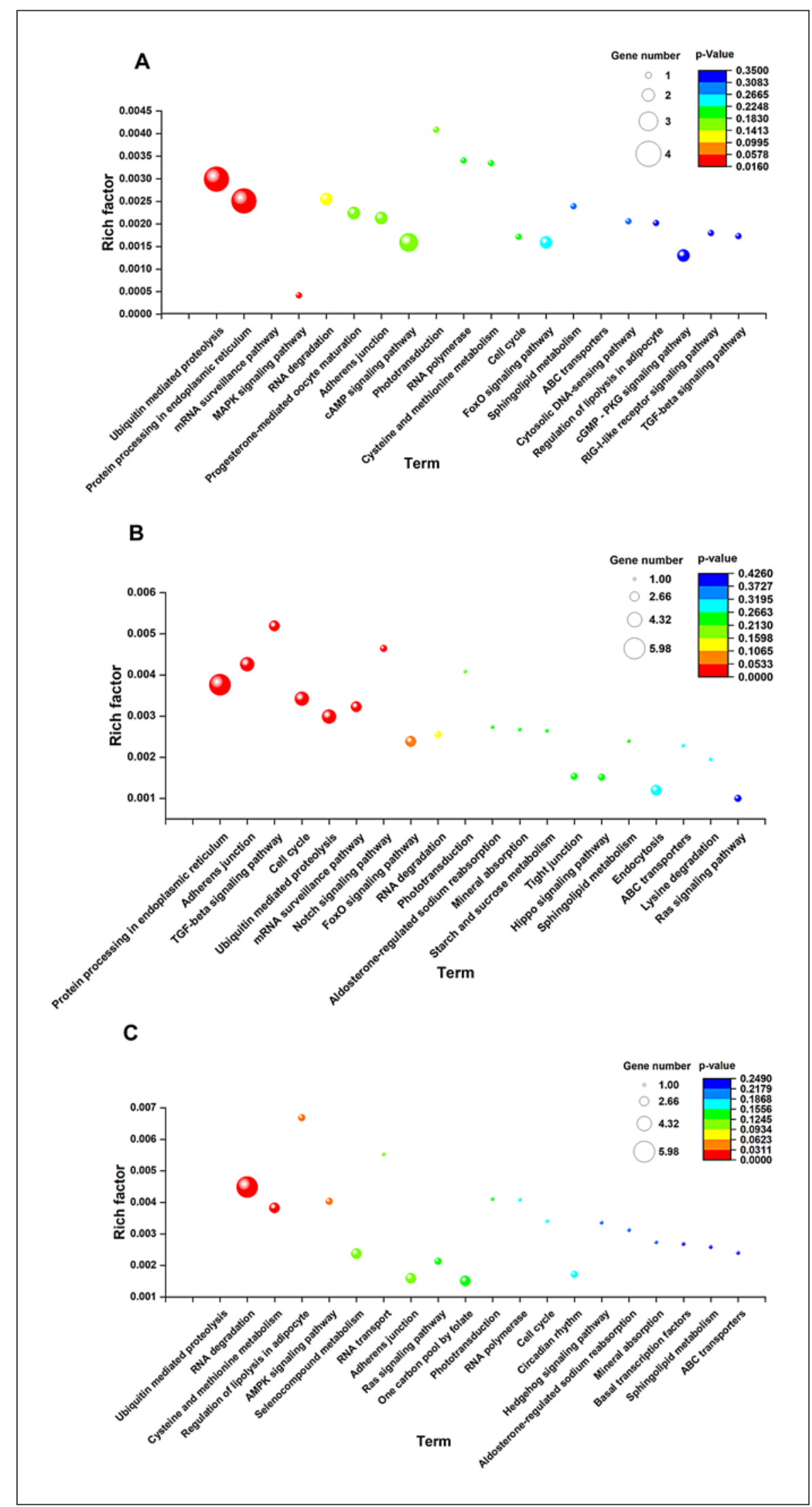



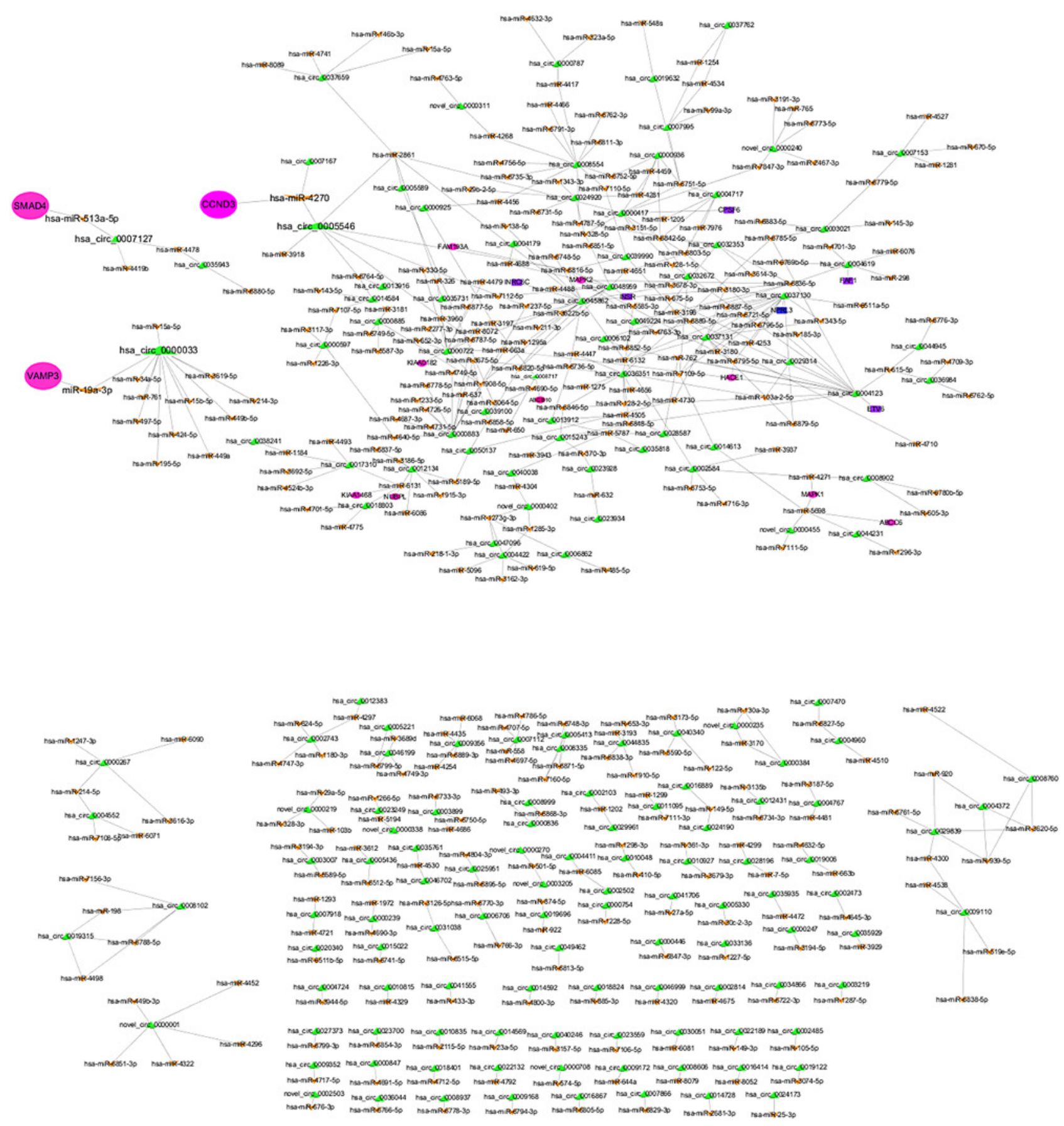

Fig. 4. mRNA-circRNA-miRNA network. Cytoscape was used to generate a circRNA-miRNA co-expression network. The network consists all of differentially expressed circRNAs in three groups (day 20 vs. day 0 , day 40 vs. day 20, day 40 vs. day 0 ), their corresponding predicted target miRNAs and some related mRNAs. Green triangle: circRNA; orange V line node: miRNA; blue rectangle: parental gene of the circRNA; purple ellipse: miRNA target gene. Nodes indicate the number of miRNA, indirectly representing circRNA function.

linked to storage lesion events, circRNAs sponged with miRNAs, and miRNAs can target with mRNAs. These miRNAs or mRNAs were involved in the cell apoptosis.

The expression of these circRNAs and their parental gene enrichment pathways were as follows. In day 20 ver- sus day 0 and day 40 versus day 0 , circRNAs including hsa_circ_0004619, hsa_circ_0000619, hsa_circ_0000446 (MAPK signaling pathway), hsa_circ_0000722, hsa circ_0000754 (regulation of actin cytoskeleton), hsa circ_0006717 (mRNA surveillance), and hsa 
circ_0008554 were decreased, while the other circRNAs like hsa_circ_0000847 (adherens junction and cell cycle) was increased. CircRNAs such as hsa_circ_0007127 (RNA degradation), hsa_circ_0007112 (ubiquitin-mediated proteolysis), novel_circ_0000311 (cysteine and methionine metabolism pathway), hsa_circ_0000033, hsa circ_0049462 (protein processing in endoplasmic reticulum), and hsa_circ_0005546 (cysteine and methionine metabolism pathway) were decreased in day 20 versus day 0 , but increased in day 40 versus day 0 . The circRNAs like hsa_circ_0000739 (RNA transport) had no change in day 20 versus day 0 , but it was decreased in day 40 versus day 20. The circRNAs like hsa_circ_0004724 (ubiquitinmediated proteolysis) was only decreased in day 40 versus day 0, while the circRNAs like hsa_circ_0031038 (TGF- $\beta$ and cell cycle) was only increased in day 40 versus day 0 (Fig. 5A).

Experimental Validation of Four Apoptosis-Associated circRNA-miRNA-mRNA Axes

Generally, according to CeRNA mechanism, thus is the binding capacity of circRNA on miRNA and miRNA on mRNA, the variation of circRNA and mRNA should be in the same orientation, while the variation of circRNA and miRNA should be in opposite orientation. We predicted the downstream miRNAs of significantly increased circRNAs and the upstream miRNAs of significantly decreased mRNAs. We selected 3 DE-circRNADE-miRNA interactions related with apoptosis from the identified 15 DE-circRNA-DE-miRNA interactions for verification. Thereafter, those miRNAs target mRNA and miRNAs were verified through RT-qPCR (Fig. 5). Finally, 3 circRNA-miRNA-mRNA axes were constructed, which include hsa_circ_0007127-miR-513a-5pSMAD4, hsa_circ_0000033-miR-19a-3p-VAMP3, and hsa_circ_0005546-miR-4720-CCND3. These axes were enlarged and shown by cytoscape software (Fig. 4).

\section{Discussion}

In vitro storage RBCs undergo a series of changes, such as oxidation, glycosylation, ubiquitination, and amidation, triggering programmed death, which are also referred to as eryptosis [7]. Recently, the upregulated miR-570-3p in platelets target with ATP5L mRNA seems close to ATP loss in ex vivo, which supports that non-coding RNA may play an important role in nonnucleated cells [8]. Notably, during the entire RBC storage period, almost $70 \%$ DE-circRNA expression followed the same trend (up or down), while $30 \%$ circRNAs showed opposite trends (day 20 vs. day 0 and day 40 vs. day 20). Since there is no transcription in RBC, the high expression level of circRNAs should be due to the dif- ferential degradation/decay. Thus, the slow or fast degradation/decay rate of gene in differential storage time might cause the increase or decrease of circRNAs generation. Furthermore, the increase of endogenous circRNA levels depends on depleting a variety of core spliceosomal components, inhibition of $3^{\prime}$ end processing and transcription termination [9]. Importantly, we found a possible explanation for the circRNA increase after 20 days as follows. Some different circular RNAs from the same parental gene had opposite expression trend after 20 days, and our data suggest that a part of circRNAs biogenesis from the same parental gene may have competitive relationship. For example, the hsa circ_0000033 increase after 20 days may be due to the decrease of hsa_circ_0010972, novel_circ_0000090, and novel_circ_0002222 after 20 days. In the sequencing results, only these four circRNAs are back splicing from ENST00000476272. Furthermore, during the development of human embryos, nucleic acid substances continue to increase, but some circRNA showed a decreased expression, while many circRNA showed wavy line expression during development [10]. In addition, increase trend of circRNAs also has been found in maturation of hematopoietic stem cells [11], the decrease in circRNA expression in this study may be attributed to the degradation upon long storage.

Recently, it has been reported that circRNAs were enriched in human platelets, leukocyte, and RBC, 6,228 circRNAs have been profiled in healthy human leukocytes [12]. Importantly, our high-throughput sequencing data revealed that a total of 8,802 circRNAs existed, including 2,586 known and 6,216 putative novel circRNAs. Currently, most of these circRNAs functions have not been reported, but there are still some circRNAs functions that have been discovered as follows. (i) Knockdown of circABCB10 (hsa_circ_0008717), which was increased on day 40, suppresses proliferation and enhances apoptosis in breast cancer carcinogenesis [13]. (ii) Circ-KIAA0182 (hsa_circ_0000722), which decreased on day 20, was enriched in extracellular vesicles and has the function for signaling communication from cell to cell [14]. (iii) hsa circ_0004619, which showed lower expression on day 20, participates in cancer-related pathways [15]. (iv) hsa circ_100219 derived from Fas associated factor 1 (FAF1) could initiate apoptosis and reduce the migration of cancer cells [16].

Differentially expressed circRNAs of parental genes were enriched in different pathways related to storage lesion, which is elaborated by selected ubiquitin-mediated proteolytic pathway and TGF- $\beta$ pathway. It is known that the ubiquitin-proteasome pathway is the main cytoplasmic proteolytic mechanism for selective degradation of various damaged proteins [17]. Therefore, hsa circ_0007112 (circ-HERC1), which decreased on day 20,
Zhang/Huang/Yuan/Zhang/Chen/Huang/ Li/Liu/Zhong/Huang/Huang/Wei 
Fig. 5. Expression of genes validated by RTqPCR in stored RBCs (mean \pm SD, $n=5$ ) $\left({ }^{*} p<0.05,{ }^{* *} p<0.01,{ }^{* * *} p<0.001\right)$. A Expression of circRNAs validated by RT-qPCR in stored RBCs. B Expression of selected miRNA validated by RT-qPCR in stored RBCs. C Expression of selected mRNA validated by RT-qPCR in stored RBCs.
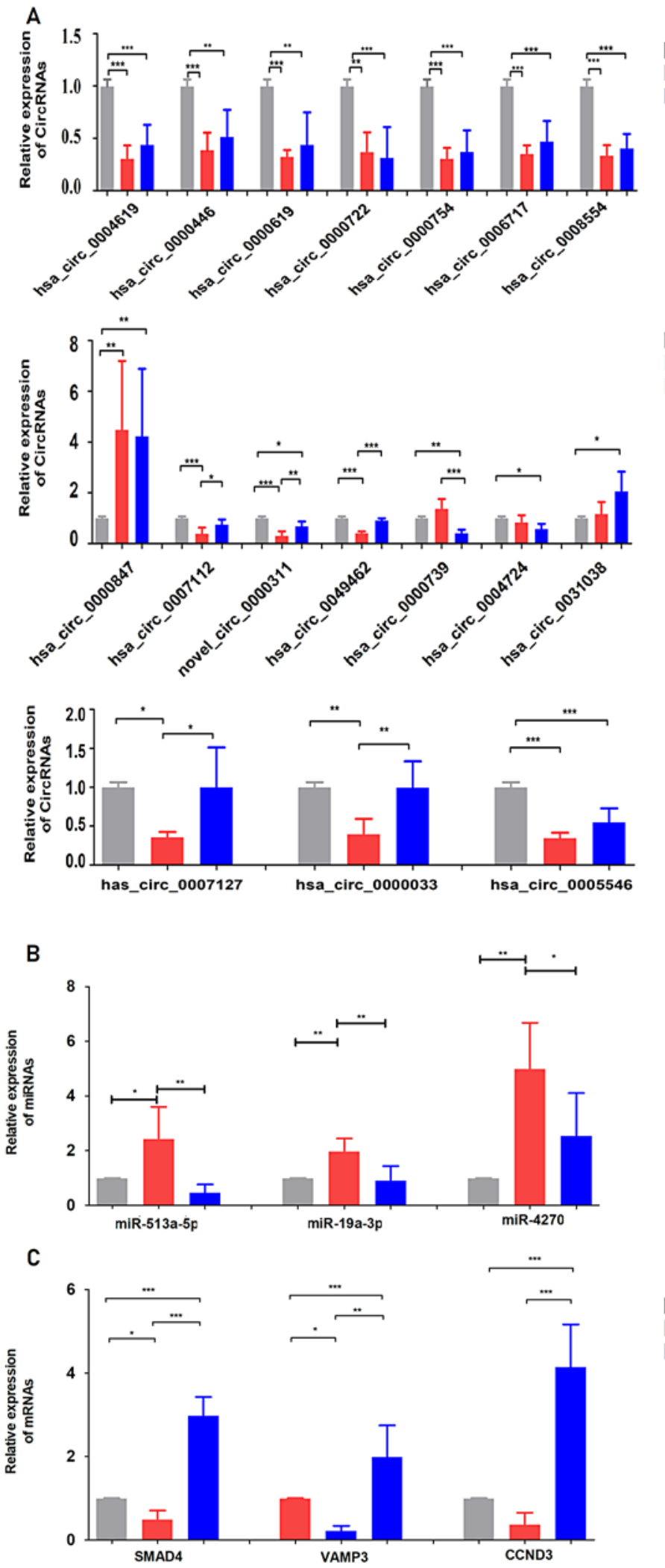

Day0

Day20

Day40 
and hsa_circ_0004724 (circ-TRIM37), which decreased on day 40, may together play an important role in the ubiquitin-mediated proteolytic pathway.

Both HERC1 and TRIM37 were complex multidomain enzymes in the E3 ubiquitin ligase. HERC1 stimulates guanine nucleotide exchanges for ARF1 and Rab proteins and may be involved in membrane transport during apoptosis [18]. TGF- $\beta$ is an important cytokine that regulates tissue inflammation and repair, and contains a large family of multifunctional cytokines that regulate cell growth, proliferation, differentiation, migration, and apoptosis [19]. hsa_circ_0000847 (circSMAD2), which increased on day 20 and day 40, would play a very important role in TGF- $\beta$ pathway. SMAD2 is a star molecule in TGF- $\beta$ pathway and could indirectly affect apoptosis and MAPK signaling pathway. hsa circ_0031038 (circ-TFDP1), which increased only on day 40 , might be a biomarker to RBC storage lesion. TFDP1 can indirectly affect the cell cycle by promoting the expression of $\mathrm{c}-\mathrm{Myc}$ and suppressing the expression of the gene $p 15$ [20]. The hypothesis we proposed for circRNA expression changes might be closely related to the storage lesion related proteins, which also requires further indepth study to double confirm.

In this study, we not only provided large-scale circRNA profiles during RBCs storage, but also experimentally characterized 3 circRNA-miRNA-mRNA regulator axes (i.e., the has_circ_0007127-miR-513a-5p-SMAD4 axis) related to storage lesion events (i.e., apoptosis) by RT-qPCR on day 0 , day 20 , and day 40 . We preliminarily confirmed that the expression of SMAD4 genes was regulated by has_circ_0007127 via sponging miR-513a-5p, and VAMP3 genes were regulated by hsa_circ_0000033 via sponging miR-19a-3p, and CCND3 genes were regulated by hsa_circ_0005546 via sponging miR-4720 in in vitro stored RBCs. Although none of the verified 3 circRNA-miRNA-mRNA regulator axes have been reported previously, several molecules were involved in certain pathophysiologic processes when separately analyzed. For instance, according to many reports, overexpressed miR-513a-5p induced significant increase in apoptosis [21-23], has-miR-19a-3p is closely related to the AMPK signaling pathway [24]. MiR-4270 was significantly increased, which may participate in oxidative stress by regulating PPARGC1A, AKT3, NOX5, PIK3C3, and WNT1 in sepsis-induced acute kidney injury [25]. During aging of human RBCs, a part of the membrane skeleton is probably lost together with some lipid bilayers, which is in good agreement with many literatures that VAMP3 mediates vesicle-vesicle fusion processes in the endosomal pathway [26]. The decrease of VAMP3 expression arrests $\mathrm{Na}^{+} / \mathrm{K}^{+} / 2 \mathrm{Cl}^{-}$co-transporter to reach to the membrane and significantly reduces the $\mathrm{Na}^{+}$absorption [27]. These evidences suggest that VAMP3 probably plays an impor- tant role in membrane remodeling and osmotic pressure in RBC storage. PAK2 and CCND3 can be directly targeted by miR-4779, and the knockdown of CCND3 inhibits the growth of cancer cells through cell cycle arrest and induces apoptosis [28]. SMAD4 is a critical component of the TGF- $\beta$ pathway implicated in erythropoiesis, and high expression level of SMAD4 promotes cell proliferation during erythroid differentiation and apoptosis in different types of tumor cells $[29,30]$. In summary, we conclude that through indirectly regulation of mRNA with binding to miRNA, hsa_circ_0007127 and hsa_ circ_0005546 are positively correlated with apoptosis in RBC storage lesion, while hsa_circ_0000033 is negatively correlated with apoptosis in RBC storage lesion and has a protective effect on the maintenance of normal $\mathrm{RBC}$ membrane morphology.

\section{Conclusions}

In summary, we studied circRNAs expressions during RBC storage, in which some were stable, while others demonstrated an increase or decrease trend during storage. These results suggest that some of these circRNAs probably are of great usage to be storage lesion markers of RBC in vitro. Further studies with a focus on circRNAmiRNA-mRNA regulator axes in RBCs could be conducted to unravel underlying mechanisms of the storage lesion.

\section{Statement of Ethics}

All procedures involving human participants were in keeping with the ethical standards of the Guangzhou First People's Hospital. All participants in this study offered their written informed consent, and the data were kept anonymous, and the consent was given official approval by the Guangzhou First People's Hospital Ethics Committees.

\section{Conflict of Interest Statement}

The authors do not have any commercial or other association that might pose a conflict of interest.

\section{Funding Sources}

This study was supported by Guangzhou Science and Technology Plan Project (201904010027), Key Clinical Technology Program of Guangzhou (2019ZD18), National Natural Science Foundation of China (81801474), the Science and Technology Fund of Shenzhen (JCYJ20180306172502097), and Shenzhen Longhua District Science and Technology Innovation Bureau Project (2020033). The authors are grateful to Shenzhen Longhua Central Hospital for financial support. DOI: $10.1159 / 000519626$
Zhang/Huang/Yuan/Zhang/Chen/Huang/ Li/Liu/Zhong/Huang/Huang/Wei 


\section{Author Contributions}

Yiyu Zhang and Yaming Wei helped with substantial contributions to the conception and design of the work; the acquisition, analysis, software processing of the data for the work; drafting the work; writing and editing the manuscript. Guoqing Huang helped with substantial contributions to the conception and design of the work; the acquisition, analysis, and interpretation of the data for the work; drafting the work; and writing the manuscript. Zhaohu Yuan and Yonggang Zhang helped with substantial contributions to the conception and design of the work; the validating, analysis, and interpretation of the data for the work; drafting the work; and writing the manuscript. Xiaojie Chen and Jianyun Huang helped with substantial contributions to data curation and writing the manuscript. Nan Li, Zhen Liu, Weijie Zhong, Huikang Huang, and Canze Huang helped with contributions to resources-supporting and writing the manuscript.

\section{Availability of Data and Materials}

All data and materials are fully available and are shown within the manuscript.

\section{References}

1 Flatt JF, Bawazir WM, Bruce LJ. The involvement of cation leaks in the storage lesion of red blood cells. Front Physiol. 2014;5:214.

2 Parthasarathi K, Lipowsky HH. Capillary recruitment in response to tissue hypoxia and its dependence on red blood cell deformability. Am J Physiol. 1999 Dec;277(6):H2145-57.

3 Chen X, Xie X, Xing Y, Yang X, Yuan Z, Wei Y. MicroRNA Dysregulation Associated with Red Blood Cell Storage. Transfus Med Hemother. 2018 Nov;45(6):397-402.

4 Jeck WR, Sharpless NE. Detecting and characterizing circular RNAs. Nat Biotechnol. 2014 May;32(5):453-61.

5 Alhasan AA, Izuogu OG, Al-Balool HH, Steyn JS, Evans A, Colzani M, et al. Circular RNA enrichment in platelets is a signature of transcriptome degradation. Blood. 2016 Mar 3;127(9):e1-e11.

6 Erman H, Aksu U, Belce A, Atukeren P, Uzun $\mathrm{D}$, Cebe T, et al. Gender and chronological age affect erythrocyte membrane oxidative indices in citrate phosphate dextrose adenine-formula 1 (CPDA-1) blood bank storage condition. Gen Physiol Biophys. 2016 Jul;35(3): 343-51.

7 Yoshida T, Prudent M, D’Alessandro A. Red blood cell storage lesion: causes and potential clinical consequences. Blood Transfus. 2019 Jan;17(1):27-52.

8 Dahiya N, Sarachana T, Kulkarni S, Wood WH III, Zhang Y, Becker KG, et al. miR-570 interacts with mitochondrial ATPase subunit $\mathrm{g}$ (ATP5L) encoding mRNA in stored platelets. Platelets. 2017 Jan;28(1):74-81.

9 Liang D, Tatomer DC, Luo Z, Wu H, Yang L, Chen LL, et al. The Output of Protein-Coding Genes Shifts to Circular RNAs When the PremRNA Processing Machinery Is Limiting. Mol Cell. 2017 Dec 7;68(5):940-e3.

10 Dang Y, Yan L, Hu B, Fan X, Ren Y, Li R, et al. Tracing the expression of circular RNAs in human pre-implantation embryos. Genome Biol. 2016 Jun 17;17(1):130.

11 Nicolet BP, Engels S, Aglialoro F, van den Akker E, von Lindern M, Wolkers MC. Circular RNA expression in human hematopoietic cells is widespread and cell-type specific. Nucleic Acids Res. 2018 Sep 19;46(16):8168-80.
12 Gaffo E, Boldrin E, Dal Molin A, Bresolin S, Bonizzato A, Trentin L, et al. Circular RNA differential expression in blood cell populations and exploration of circRNA deregulation in pediatric acute lymphoblastic leukemia. Sci Rep. 2019 Oct 11;9(1):14670.

13 Liang HF, Zhang XZ, Liu BG, Jia GT, Li WL. Circular RNA circ-ABCB10 promotes breast cancer proliferation and progression through sponging miR-1271. Am J Cancer Res. 2017; $7(7): 1566-76$

14 Lasda E, Parker R. Circular RNAs Co-Precipitate with Extracellular Vesicles: A Possible Mechanism for circRNA Clearance. PloS one. 2016;11(2):e0148407.

15 Lü L, Sun J, Shi P, Kong W, Xu K, He B, et al. Identification of circular RNAs as a promising new class of diagnostic biomarkers for human breast cancer. Oncotarget. 2017 Jul 4; 8(27):44096-107.

16 Zhang L, Zhou F, Li Y, Drabsch Y, Zhang J, van Dam H, et al. Fas-associated factor 1 is a scaffold protein that promotes $\beta$-transducin repeat-containing protein $(\beta-\operatorname{TrCP})$ mediated $\beta$-catenin ubiquitination and degradation. The Journal of biological chemistry. 2012 Aug 31;287(36):30701-10.

17 Spratt DE, Walden H, Shaw GS. RBR E3 ubiquitin ligases: new structures, new insights, new questions. Biochem J. 2014 Mar 15; 458(3):421-37.

18 Bachmayr-Heyda A, Reiner AT, Auer K, Sukhbaatar N, Aust S, Bachleitner-Hofmann $\mathrm{T}$, et al. Correlation of circular RNA abundance with proliferation--exemplified with colorectal and ovarian cancer, idiopathic lung fibrosis, and normal human tissues. Sci Rep. 2015 Jan 27;5:8057.

19 Finnson KW, McLean S, Di Guglielmo GM, Philip A. Dynamics of Transforming Growth Factor Beta Signaling in Wound Healing and Scarring. Adv Wound Care (New Rochelle). 2013 Jun;2(5):195-214.

20 Nass SJ, Li M, Amundadottir LT, Furth PA, Dickson RB. Role for Bcl-xL in the regulation of apoptosis by EGF and TGF beta 1 in c-myc overexpressing mammary epithelial cells. Biochem Biophys Res Commun. 1996 Oct 3; 227(1):248-56.
21 Shin S, Moon KC, Park KU, Ha E. MicroRNA-513a-5p mediates TNF- $\alpha$ and LPS induced apoptosis via downregulation of $\mathrm{X}$ linked inhibitor of apoptotic protein in endothelial cells. Biochimie. 2012 Jun;94(6): 1431-6.

22 Dong X, Xu M, Ren Z, Gu J, Lu M, Lu Q, et al. Regulation of CBL and ESR1 expression by microRNA-223p, 513a-5p and 625-5p may impact the pathogenesis of dust mite-induced pediatric asthma. Int J Mol Med. 2016 Aug; 38(2):446-56.

23 Li S, Xu YN, Niu X, Li Z, Wang JF. miR-513a$5 \mathrm{p}$ targets $\mathrm{Bcl}-2$ to promote dichlorvos induced apoptosis in HK-2 cells. Biomed Pharmacother. 2018 Dec;108:876-82.

24 Zheng Y, Kong J, Li Q, Wang Y, Li J. Role of miRNAs in skeletal muscle aging. Clin Interv Aging. 2018;13:2407-19.

25 Ge QM, Huang CM, Zhu XY, Bian F, Pan SM. Differentially expressed miRNAs in sepsisinduced acute kidney injury target oxidative stress and mitochondrial dysfunction pathways. PloS one. 2017;12(3):e 0173292.

26 Ciana A, Achilli C, Gaur A, Minetti G. Membrane Remodelling and Vesicle Formation During Ageing of Human Red Blood Cells. Cell Physiol Biochem. 2017;42(3):1127-38.

27 Caceres PS, Mendez M, Haque MZ, Ortiz PA. Vesicle-associated Membrane Protein 3 (VAMP3) Mediates Constitutive Trafficking of the Renal Co-transporter NKCC2 in Thick Ascending Limbs: ROLE IN RENAL FUNCTION AND BLOOD PRESSURE. J Biol Chem. 2016 Oct 14;291(42):22063-73.

28 Koo KH, Kwon H. MicroRNA miR-4779 suppresses tumor growth by inducing apoptosis and cell cycle arrest through direct targeting of PAK2 and CCND3. Cell Death Dis. 2018 Jan 23;9(2):77.

29 Doss JF, Corcoran DL, Jima DD, Telen MJ, Dave SS, Chi JT. A comprehensive joint analysis of the long and short RNA transcriptomes of human erythrocytes. BMC genomics. 2015 Nov 16;16:952.

30 Li X, Li X, Lv X, Xiao J, Liu B, Zhang Y. Smad4 Inhibits VEGF-A and VEGF-C Expressions via Enhancing Smad3 Phosphorylation in Colon Cancer. Anat Rec (Hoboken). 2017 Sep; 300(9):1560-9. 